Article

\title{
Inhibition of adipogenesis in mouse 3T3-L1 pre- adipocytes by an extract from Sophora japonica fruit acting via the AMP-activated protein kinase pathway
}

\author{
Cheol Park ${ }^{1}$, Young-Kyung Lee ${ }^{2}$, Chul Hwan Kim ${ }^{2}$, Su Young Shin ${ }^{2}$, Kyung-Min Choi ${ }^{2}$, In-Jun \\ Yang ${ }^{3}$, Gi-Young Kim ${ }^{4}$, Yung Hyun Choi ${ }^{5, *}$ and Jin-Woo Jeong ${ }^{2, *}$ \\ 1 Division of Basic Sciences, College of Liberal Studies, Dong-eui University, Busan, 47340, Republic of \\ Korea; parkch@deu.ac.kr (C.P.) \\ 2 Nakdonggang National Institute of Biological Resources, 137, Donam 2-gil, Sangju-si, Gyeongsangbuk-do \\ 37242, Republic of Korea; leeyk@nnibr.re.kr (Y.-K.L.); kchul1204@nnibr.re.kr (C.H.K.); ssy337@nnibr.re.kr \\ (S.Y.S.); kyungmc69@nnibr.re.kr (K.-M.C.) \\ 3 Department of Physiology, College of Oriental Medicine, Dongguk University, Gyeongju 780-714, Republic \\ of Korea; injuny@dongguk.ac.kr (I.-J.Y.) \\ 4 Laboratory of Immunobiology, Department of Marine Life Sciences, Jeju National University, Jeju 63243, \\ Republic of Korea; immunkim@jejunu.ac.kr (G.-Y.K.) \\ 5 Department of Biochemistry, Dong-eui University College of Korean Medicine, Busan 47227, Republic of \\ Korea \\ * Correspondence: choiyh@deu.ac.kr (Y.H.C.); jwjeong@nnibr.re.kr (J.-W.J.); Tel.: +82-51-850-7421 (Y.H.C.); \\ +82-54-530-0884 (J.-W.J.)
}

\begin{abstract}
Background: The world-wide rate of obesity is increasing continuously, representing a serious medical threat since it is associated with a variety of diseases including type 2 diabetes, cardiovascular disease, and numerous cancers. Sophora japonica is used as a traditional herb for medicinal purposes in eastern Asia. However, the anti-obesity effects of S. japonica fruit have not been explored. Objective: The aim of this study is to investigate the inhibition of adipocyte differentiation and adipogenesis by an ethanol extract of $S$. japonica fruit (EESF) in 3T3-L1 preadipocytes. Methods: MTT assay, Oil Red O staining, and Triglyceride contents were used to investigate lipid accumulation in 3T3-L1 cells to clarify adipocyte differentiation. The expression levels of various molecular signals associated with adipogenesis and lipogenesis were examined by western blot analysis. Results: Our results demonstrate that EESF suppressed the terminal differentiation of 3T3-L1 pre-adipocytes in a dose-dependent manner, as confirmed by a decrease in lipid droplet number and lipid content through Oil Red O staining. EESF significantly reduced the accumulation of cellular triglyceride, which was associated with a significant inhibition of the levels of pro-adipogenic transcription factors, downregulated the expression levels of adipocytespecific proteins. Furthermore, EESF treatment effectively increased the phosphorylation of AMPK and ACC. Conclusions: These results together indicate that EESF has significant effects on the inhibition of adipogenesis and acts by stimulating the AMPK signaling pathway. Further studies will be needed to identify the active compounds in S. japonica.
\end{abstract}

Keywords: Sophora japonica, Adipogenesis, AMPK, 3T3-L1 pre-adipocytes

\section{Introduction}

Obesity is a metabolic disorder that is caused by an abnormal regulation of energy metabolism in the body when energy intake exceeds consumption for a prolonged period of time. Under these conditions the excess energy is stored as fat that accumulates in subcutaneous or abdominal regions in the body. Obesity is a known risk factor for diabetes, cardiovascular diseases, neurodegenerative 
diseases such Parkinson's and Alzheimer's, as well as of various type of cancer including breast, endometrial, gastric, colorectal, and esophageal cancer. Of particular importance in these disease states are the adipokines produced and secreted by adipocytes [1-3]. According to a survey conducted by the World Health Organization (WHO), the global obesity rate in 2014 was 39\%, a two-fold increase since 1980. In line with this, the obesity rate in Korea is reported to have steadily increased from an average of $29.2 \%$ in 2001 to $32.9 \%$ in 2014 due to a westernization of dietary habits and changes in living conditions. Obesity is thus receiving growing attention as a grave health problem $[4,5]$. Treatment of obesity generally involves drug administration and surgery in addition to improvements in lifestyle such as dietary control and exercise. The two most widely used drugs for obesity treatment, Sibutramine and Orlistat, cause not only cardiovascular and gastrointestinal disorders but also headache, severe thirst, constipation, insomnia, and palpitations. Therefore, the development of a therapeutic agent with proven safety and effectiveness is urgently needed $[6,7]$. One possible approach to this problem is through the identification of natural products with antiobesity effects.

In general, obesity is known to be caused by two critical factors including adipocyte hypertrophy as a result of the triglyceride accumulation induced by adipogenesis, and adipocyte hyperplasia caused by the proliferation and differentiation of adipocytes [8]. Adipogenesis is the differentiation process by which adipocytes are generated from fibroblastic pre-adipocytes, and is accompanied by changes in cell morphology, gene expression, and hormone sensitivity. Adipogenesis is known to be sequentially influenced by adipogenic transcription factors including sterol response element binding protein 1c (SREBP1c), peroxisome proliferator-activated receptor $\gamma$ (PPAR $\gamma)$, and cytidinecytidine-adenosine-adenosine-thymidine (CCAAT)/enhancer binding proteins (C/EBPs) $[9,10]$. In addition, once adipocytes have been formed through adipogenesis, not only morphological characteristics, such as the triglyceride accumulation seen in white adipocytes, but also the expression of adipocyte-specific genes including adipocyte-specific lipid binding protein (aP2) and leptin is known to occur [11]. AMP-activated protein kinase (AMPK), in particular, is a key regulator of energy homeostasis that is known to control adipogenesis, and consequently has been studied as a drug target for obesity prevention and treatment [12]. The regulation of adipogenesis could be used as an important process to inhibit the inhibition of obesity since it is an essential mechanism that leads to adipocyte production.

Sophora japonica from the Fabaceae family is commonly used as a plant feedstock for the production of flavonoids. This plant is widespread in Asia and is also cultivated in the southern regions of Russia. Its buds, dried flowers, and fruit are medicinal herbs that are used in traditional Korean medicine particularly the dried fruit referred to as Sophorae Fructus [13]. Numerous biological activities of extracts, fractions, and single compounds derived from S. japonica fruits have been reported, such as anti-osteoporosis effects, anti-inflammatory effects, anti-tumor effects, reductions in menopausal symptoms, and hemostatic effects [14-20]. Although functional substances with various pharmacological activities have been reported, studies on their anti-obesity effects and the mechanisms underlying these effects are relatively very rare.

The present study thus investigated the anti-obesity effects of an ethanol extract of Sophora japonica fruit (EESF) and how EESF influences adipogenesis, and reports significant findings.

\section{Materials and Methods}

\subsection{Materials}

The insulin, dexamethasone, and IBMX used for the differentiation of adipocytes, the Oil Red O used to detect triglyceride formation in differentiated adipocytes, and compound $\mathrm{C}$, which was used as an AMPK inhibitor, were all purchased from Sigma-Aldrich (St. Louis, MO, USA). The primary antibodies used in the protein analysis were purchased from either Santa Cruz Biotechnology Inc. (Santa Cruz, CA, USA) or Cell Signaling Technology (Beverly, MA, USA) (Table 1), and the secondary antibodies used in the immunoblotting: peroxidase-labeled donkey anti-rabbit and peroxidase- 
labeled sheep anti-mouse immunoglobulin, were purchased from Amersham Life Science Corp. (Arlington Heights, IL, USA).

Table 1. Antibodies used in the present study

\begin{tabular}{cclc}
\hline Antibody & Origin & \multicolumn{1}{c}{ Company } & Catalogue No. \\
\hline PPAR $\gamma$ & Rabbit polyclonal & Santa Cruz Biotechnology, Inc. & SC-7196 \\
C/EBP $\alpha$ & Rabbit polyclonal & Cell Signaling Technology, Inc. & 2295 \\
C/EBP $\beta$ & Rabbit polyclonal & Cell Signaling Technology, Inc. & 3087 \\
aP2 & Goat polyclonal & Santa Cruz Biotechnology, Inc. & SC-18661 \\
Leptin & Rabbit polyclonal & Santa Cruz Biotechnology, Inc. & SC-842 \\
AMPK & Rabbit polyclonal & Santa Cruz Biotechnology, Inc. & SC-25792 \\
pAMPK & Rabbit polyclonal & Cell Signaling Technology, Inc. & 2535 \\
ACC & Rabbit polyclonal & Santa Cruz Biotechnology, Inc. & SC-30212 \\
pACC & Rabbit polyclonal & Cell Signaling Technology, Inc. & 3661 \\
Actin & Mouse monoclonal & Santa Cruz Biotechnology, Inc. & SC-47778 \\
\hline
\end{tabular}

\subsection{Preparation of the ethanol extract from Sophora japonica fruit (EESF)}

To prepare the ethanol extract of $S$. japonica fruit (EESF), the S. japonica fruit was washed thoroughly in running water, dried, and finely ground. After adding 2,000 $\mathrm{mL}$ of distilled water per $100 \mathrm{~g}$ of $\mathrm{S}$. japonica fruit, the mixture was boiled at $110^{\circ} \mathrm{C}$ for $2 \mathrm{~h}$ in a heater equipped with a reflux cooling device. Next, after centrifuging at 3,000 rpm for $20 \mathrm{~min}$, and removing the residue and filtering the supernatant using a Whatman filter (No. 2), a solid extract was produced through a decompression evaporation process. The solid component was then pulverized using a mortar and pestle and stored at $-70^{\circ} \mathrm{C}$. A stock solution of EESF was made to $100 \mathrm{mg} / \mathrm{mL}$ using dimethyl sulfoxide (DMSO) as solvent and used to prepare dilutions for experimentation.

\subsection{Cell culture}

The mouse fibroblast cell line 3T3-L1 pre-adipocytes used in this study was obtained from American Type Culture Collection (ATCC, Manassas, VA, USA). 3T3-L1 pre-adipocytes were cultured using growth medium containing 90\% Dulbecco's Modified Eagle's Medium (DMEM, Gibco BRL, Grand Island, NY, USA), 10\% bovine calf serum (BCS, Gibco BRL, Grand Island, NY, USA) and $1 \%$ penicillin and streptomycin (Gibco BRL) at $37^{\circ} \mathrm{C}$ in an atmosphere of $5 \% \mathrm{CO}_{2}$. The growth medium was replaced every $48 \mathrm{~h}$ to prevent overgrowth of cells.

\subsection{T3-L1 pre-adipocyte differentiation induction and morphological observation}

Prior to differentiation, 3T3-L1 pre-adipocytes were maintained in a growth medium containing $10 \%$ BCS and $1 \%$ penicillin and streptomycin until they reached confluency. Confluent cells were then cultured using differentiation medium containing $10 \%$ fetal bovine serum (FBS, Gibco BRL) and $1 \%$ penicillin and streptomycin for a further two days, after which the medium was replaced with the one containing $10 \mu \mathrm{g} / \mathrm{mL}$ insulin, $1 \mu \mathrm{M}$ dexamethasone, and $0.5 \mu \mathrm{M}$ IBMX (MDI) for a further two days. Afterwards, the medium was replaced with one containing $10 \mu \mathrm{g} / \mathrm{mL}$ insulin every two days. In addition, to assess inhibition of 3T3-L1 pre-adipocyte differentiation, cells were treated with EESF by replacing the medium with one containing insulin and MDI. The 3T3-L1 pre-adipocytes whose differentiation had been induced as described, were subsequently used in various experimental analyses. To examine the effects of EESF on the morphological changes caused by differentiation of 3T3-L1 pre-adipocytes into adipocytes, the same methods described above were used for differentiation and EESF treatment, after which the cells were observed under $\times 200$ magnification using an inverted microscope (Carl Zeiss, Gottingen, Germany). Images were taken using the Axio Vision program.

\subsection{Cell viability measurement}


To examine the effects of EESF on the cell viability of 3T3-L1 pre-adipocytes, the cells were plated into a 6-well culture plate and cultured until confluent. The confluent cells were then treated with EESF. After $72 \mathrm{~h}$, the supernatant was removed, and the cells were treated with $0.05 \%$ trypsin-EDTA to allow for their detachment from the plate. Next, phosphate-buffered saline (PBS) and a $0.5 \%$ trypan blue solution (Gibco BRL) were added to each well. After approximately two minutes, a hemocytometer was used to count the live cells in the sample under an inverted microscope and the relative cell counts were compared. As an alternative way to measure cell viability, the cells were prepared as described above, then after removing the medium, $0.5 \mathrm{mg} / \mathrm{mL}$ tetrazolium bromide salt (MTT, Amresco, Solon, OH, USA) was added and the cells were cultured in complete darkness in a $\mathrm{CO}_{2}$ incubator. After $3 \mathrm{~h}$, the MTT reagent was removed and DMSO was added to the wells to completely dissolve the formazan produced. The dissolved formazan $(200 \mu \mathrm{L})$ was then transferred to a 96-well plate and the OD at $540 \mathrm{~nm}$ measured using ELISA reader (Molecular Devices, Sunnyvale, CA, USA).

\subsection{Oil Red $O$ staining and triglyceride quantification}

Oil red O staining is a dye binding method used to quantify total lipids. The lipid-specific binding allows for a quantitative analysis of the lipids produced by adipocytes. Oil red $\mathrm{O}$ staining was carried out to examine the effects of EESF on the production of lipid droplets inside 3T3-L1 adipocytes following induction of differentiation. For both control and 3T3-L1 adipocytes treated with varying concentrations of EESF, the medium was removed and the cells were washed with PBS then fixed for $1 \mathrm{~h}$ using 3.7\% formalin. After fixing, the cells were washed with $60 \%$ isopropanol and treated with the Oil Red O solution at room temperature for $20 \mathrm{~min}$ to stain the lipid droplets. After the completion of staining, the Oil Red O solution was removed and the cells were washed three times with distilled water. The stained cells were then observed under an inverted microscope, and images were taken using the Axio Vision program. In addition, for a quantitative analysis of the level of inhibition on EESF-induced triglyceride production, the cells were treated with $100 \%$ isopropanol to dissolve the Oil Red $\mathrm{O}$ and $200 \mu \mathrm{L}$ of the dissolved solution was transferred to a 96-well plate after which the OD was measured at $500 \mathrm{~nm}$ using an ELISA reader.

\subsection{Western blot analysis}

A western blot analysis was used to examine the effects of EESF on the expression of adipogenic transcription factors and adipocyte-specific genes. 3T3-L1 adipocytes were prepared as previously described, after which lysis buffer [ $25 \mathrm{mM}$ Tris- $\mathrm{Cl}$ (pH 7.5), $250 \mathrm{mM} \mathrm{NaCl}, 5 \mathrm{mM}$ EDTA, 1\% NP-40, 1 $\mathrm{mM}$ phenymethylsulfonyl fluoride (PMSF), $5 \mathrm{mM}$ dithiothreitol (DTT)] was added and lysis was allowed to proceed at $4^{\circ} \mathrm{C}$ for $1 \mathrm{~h}$. The cell lysate was prepared by centrifugation at $14,000 \mathrm{rpm}$ for 30 min. The concentration of proteins in the supernatant was quantified using the Bio-Rad protein quantification agent (Bio-Rad, Hercules, CA, USA) according to the manufacturer's instructions. Lysate was mixed with an equal volume of Laemmli sample buffer (Bio-Rad) prior to electrophoresis on a sodium dodecyl sulphate (SDS)-polyacrylamide gel. The separated proteins were then transferred to a nitrocellulose membrane (Schleicher and Schuell, Keene, NH, USA) by electroblotting. The nitrocellulose membrane was then blocked with $5 \%$ skimmed milk prior to incubation with the appropriate primary antibody at $4^{\circ} \mathrm{C}$ for $12 \mathrm{~h}$. The membranes were then washed with PBS-T, and then incubated with a secondary antibody appropriate for each of the primary antibodies at room temperature for $1 \mathrm{~h}$. Immunoreactive bands were detected using an Enhanced Chemiluminescence (ECL) reagent (Amersham Life Science Corp.) and exposure to an X-ray film to allow for an analysis of the expression levels of specific proteins.

\subsection{Statistical Analysis}

All experiments were replicated in three independent experiments. All data were expressed as the mean \pm SD and analyzed using the GraphPad Prism software (version 5.03; GraphPad Software, Inc., La Jolla, CA, USA). ANOVA with Bonferroni multiple comparison test was used to confirm 
significant differences among the group means. A value of $p<0.05$ was considered to represent a statistically significant difference.

\section{Results and Discussion}

\subsection{Effects of EESF on the growth of 3T3-L1 pre-adipocytes}

To determine the cytotoxic effects of EESF on 3T3-L1 pre-adipocytes, the growth and the cell viability of 3T3-L1 pre-adipocytes were examined following EESF treatment. First, to assess growth inhibition by EESF, the cultured cells were treated with varying concentrations of EESF for $72 \mathrm{~h}$, and live cell numbers, determined using a hemocytometer, were compared (Fig. 1A). As shown, no significant growth inhibition was apparent up to $100 \mu \mathrm{g} / \mathrm{mL}$ EESF, but a dose-dependent increase in growth inhibition was observed above $120 \mu \mathrm{g} / \mathrm{mL}$. An MTT assay was used to determine the effects of EESF on the viability of 3T3-L1 pre-adipocytes under the same conditions described above (Fig. 1B). EESF did not induce a significant change in cell viability up to $100 \mu \mathrm{g} / \mathrm{mL}$ EESF but a strong inhibitory effect on cell survival was observed above $120 \mu \mathrm{g} / \mathrm{mL}$. Based on these results, EESF was determined to exhibit negligible cytotoxicity up to $100 \mu \mathrm{g} / \mathrm{mL}$; hence, subsequent experiments were performed with EESF concentrations up to $100 \mu \mathrm{g} / \mathrm{mL}$.

A)

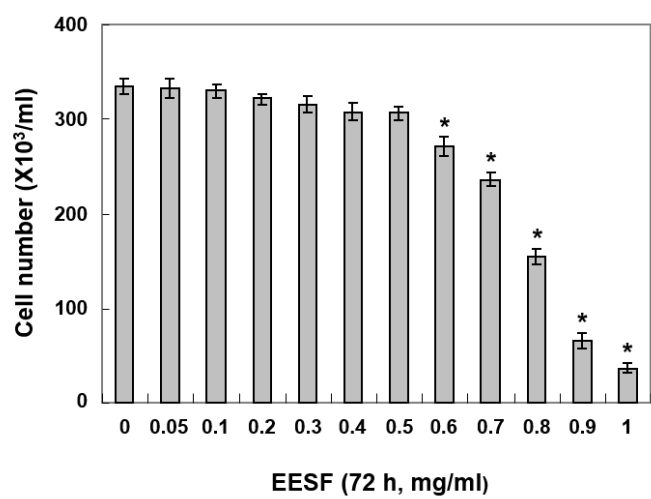

B)

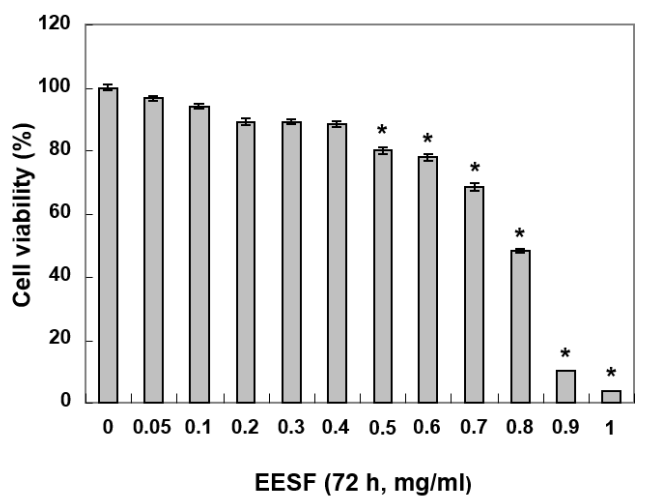

Figure 1. Effects of EESF on the growth of mouse 3T3-L1 pre-adipocytes. Cells were treated with the indicated concentrations of EESF for $72 \mathrm{~h}$. Cell number (A) and viability (B) were determined by hemocytometer counts of trypan blue excluding cells and an MTT assay, respectively. The data are expressed as the mean $\pm \mathrm{SD}$ of three independent experiments. (ANOVA: ${ }^{*} p<0.05$ vs. untreated control).

\subsection{Effects of EESF on the production of lipid droplets}

The lipid droplets produced during the process of differentiation into adipocytes are in a vesicle surrounded by a phospholipid monolayer. They begin to form when triglyceride and cholesterol ester accumulate in the bilayer of the endoplasmic reticulum so that the layers start to separate [21]. The lipid droplets in mature adipocytes are known to be controlled by the influx of triglycerides due to the action of lipoprotein lipase (LPL) and release of triglycerides mediated by adipose triglyceride lipase (ATGL) and hormone sensitive lipase (HSL). These lipid droplets have been reported to participate in the induction of obesity as well as in diseases such as cancer, arteriosclerosis, and type II diabetes [22,23]. Thus, to examine the effects of EESF on the production of lipid droplets during adipogenesis, 3T3-L1 pre-adipocytes which had been induced to differentiate were treated with EESF and the extent of intracellular production of lipid droplets was observed under an inverted microscope before and after Oil Red O staining. As shown in Fig. 2, lipid droplets were not produced when differentiation was not induced, but following MDI treatment to induce differentiation, the intracellular production of lipid droplets was readily apparent. The number of lipid droplets was substantially inhibited in a dose-dependent manner upon EESF treatment. These data indicate that EESF inhibits the differentiation of 3T3-L1 pre-adipocytes into adipocytes. 


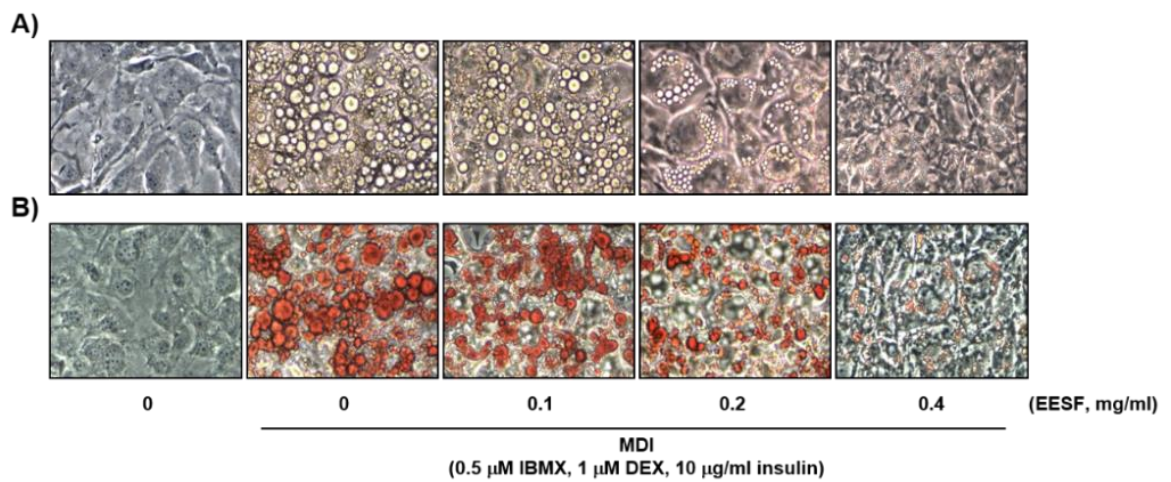

Figure 2. Effects of EESF on the microscopic morphological changes and lipid droplet accumulation in differentiated mouse 3T3-L1 adipocytes. Differentiation of confluent mouse 3T3-L1 pre-adipocytes was initiated with MDI $(0.5 \mu \mathrm{M}$ 3-isobytyl-1-methylxanthine, $1 \mu \mathrm{M}$ dexamethasone, and $10 \mu \mathrm{g} / \mathrm{mL}$ insulin) and maintained in DMEM-5\% FBS medium (maintenance differentiation medium) in the absence or presence of EESF for 8 days. (A) Differentiating 3T3-L1 cells were visualized by light microscopy. Magnification, $\times 200$. (B) Cells were fixed and stained with Oil Red O to visualize lipid droplets by light microscopy. Magnification, $\times 200$.

\subsection{Effects of EESF on the production of triglycerides}

Triglycerides are composed of one glycerol molecule connected to three fatty acid molecules via ester bonds. They account for over $95 \%$ of fat ingested as food, and along with glucose, they are used as an important energy source by cells [24]. However, when an excess amount of triglycerides is absorbed and stored by adipocytes they can contribute to obesity and thereby cause various diseases [25]. Triglyceride storage by adipocytes is known to be mediated by triglyceride-rich lipoproteins including chylomicrons and very low density lipoprotein (VLDL) [26]. Thus, to examine the effects of EESF on the production of triglycerides, the Oil Red O-stained lipid droplets were extracted using isopropanol and the triglyceride content was determined as shown in Fig. 3. During the process of MDI-induced differentiation into adipocytes, triglyceride production markedly increased; however, EESF treatment led to a gradual decrease in triglyceride production, with the maximum concentration $100 \mu \mathrm{g} / \mathrm{mL}$ EESF producing an approximately $80 \%$ inhibition of triglyceride production. The result indicates that there is an inhibitory effect of EESF on the production of triglycerides, and this is correlated with the inhibition of lipid droplet formation.

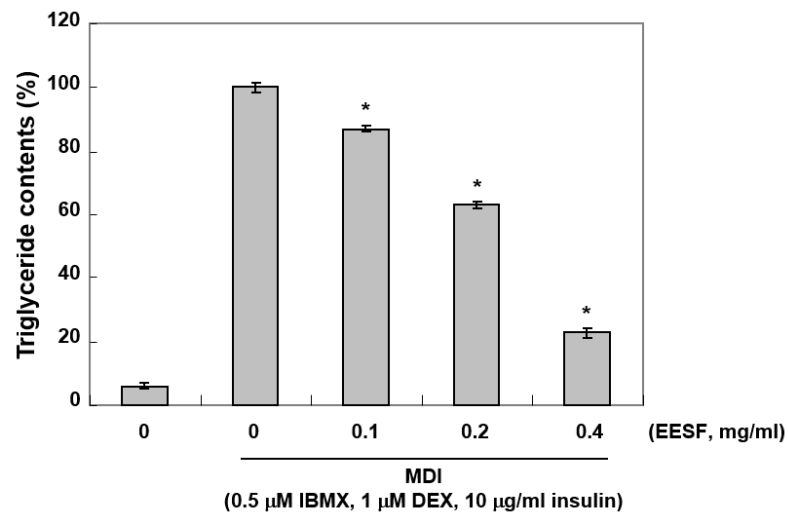

Figure 3. Inhibitory effects of EESF on triglyceride accumulation in differentiated mouse 3T3-L1 adipocytes. Triglyceride contents were determined by Oil Red $\mathrm{O}$ staining after treatment in the absence or presence of EESF. Quantification of triglyceride content was performed by measuring the absorbance at $\lambda=500 \mathrm{~nm}$ using an ELISA reader. The data are expressed as the mean $\pm \mathrm{SD}$ of three independent experiments. (ANOVA: ${ }^{*} p<0.05$ vs. untreated control). 


\subsection{Effects of EESF on the expression of adipogenic transcription factors and adipocyte-specific genes}

Myriads of adipogenic transcription factors are known to be involved in each step of adipogenesis. First, in the early stages of differentiation, the expression of C/EBP $\beta$ promotes the expression of other adipogenic transcription factors C/EBP $\alpha$ and PPAR $\gamma$ which play a part in enhancing the characteristics of mature adipocytes such as insulin-sensitive glucose uptake. SREBP1c, whose expression is induced by insulin, also plays a role in increasing PPAR $\gamma$ expression $[27,28] . \mathrm{C} / \mathrm{EBP} \alpha$ and PPAR $\gamma$ are activated by adipogenic transcription factors in the early stages of differentiation are known to be the key regulators of adipogenesis. The synergistic interaction between these two genes, through the expression of adipocyte-specific proteins including aP2 and leptin, plays an important role in the morphological changes that occur as a result of differentiation such as lipid droplet production and cell size increases [29,30]. Accordingly, we examined the effects of EESF on the expression of adipogenic transcription factors and adipocyte-specific proteins. As shown in Fig. 4A, substantially increased expression levels of PPAR $\gamma, \mathrm{C} / \mathrm{EBP} \alpha$ and C/EBP $\beta$ were observed when the differentiation was induced without EESF treatment; however, upon EESF treatment, a dose-dependent decrease in expression levels was observed. EESF treatment also significantly decreased the expression levels of the adipocyte-specific proteins aP2 and leptin, as shown in Fig. 4B. Collectively, these results indicate that EESF leads to the inhibition of adipogenesis by inhibiting the expression of adipogenic transcription factors, leading to a reduction in the production of lipid droplets and triglycerides and the expression of adipocyte-specific proteins.

A)

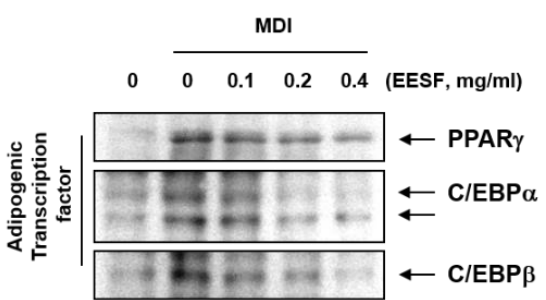

B)

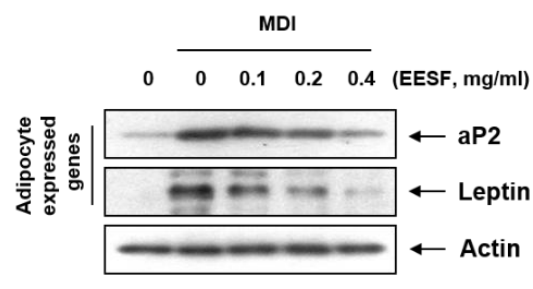

Figure 4. Effects of EESF on the levels of adipogenic transcription factors (A) and adipocyte-specific genes (B) expression in differentiated mouse 3T3-L1 adipocytes. Differentiation of confluent 3T3-L1 mouse pre-adipocytes was carried out in absence or presence of EESF for 8 days after initiation with MDI. Cells were lysed and proteins were separated by SDS-polyacrylamide gels and transferred onto nitrocellulose membranes. The membranes were probed with the indicated antibodies. Proteins were visualized using an ECL detection system. Actin was used as an internal control.

\subsection{Effects of EESF on the inhibition of adipogenesis through the AMPK signaling pathway}

AMPK, as a key regulator and energy sensor in the energy homeostasis, is known to be activated by phosphorylation caused by the increase in AMP/ATP ratio as a result of the increased intracellular AMP levels that occur when energy is consumed. AMPK can also be activated by CaMKK $\beta$ through an increase in intracellular $\mathrm{Ca}^{2+}$ levels and by LKB1, a serine/threonine protein kinase and a known tumor suppressor [31,32]. The activated AMPK increases the level of GLUT4 in muscle cells to increase glucose transport while also increasing lipid production in the body as a result of the inhibition of fatty acid synthase (FAS) and acetyl CoA carboxylase (ACC) which are both important in the synthesis of fatty acids and the inhibition of HMG-CoA reductase which limits cholesterol biosynthesis. In this way, AMPK is known to promote adipogenesis [33-35]. We therefore determined whether the AMPK signaling pathway was involved in the EESF-induced inhibition of adipogenesis. First, the expression of ACC acting on AMPK and the step downstream was examined for changes, and as shown in Fig. 5A, the phosphorylation of AMPK and ACC substantially increased. These results suggest a role for AMPK activation in the EESF-induced inhibition of adipogenesis inhibition. To address this further, the AMPK pathway was suppressed by pretreatment with compound $\mathrm{C}$, an AMPK inhibitor, and this led to an inhibition of the EESF-induced phosphorylation of AMPK and ACC, as shown in Fig. 5B, while the expression of the adipogenic transcription factors PPAR $\gamma$, 
$\mathrm{C} / \mathrm{EBP} \alpha$ and $\mathrm{C} / \mathrm{EBP} \beta$ increased. Taken together, these data indicate that there is a crucial role played by AMPK activation and the subsequent reduced expression of adipogenic transcription factors in the EESF-induced inhibition of adipogenesis. The findings of this study are expected to prove highly useful for future studies since they suggest a potential use of ethanol extracts of $S$. japonica as an antiobesity agent or drug. Nevertheless, additional biochemical addressing its potential anti-obesity mechanism and an analysis of the active compounds contained in EESF, are essential for future studies.

A)

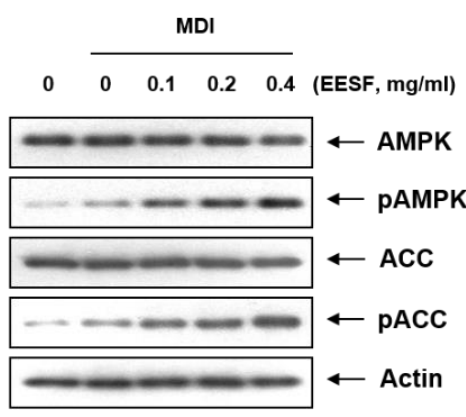

B)

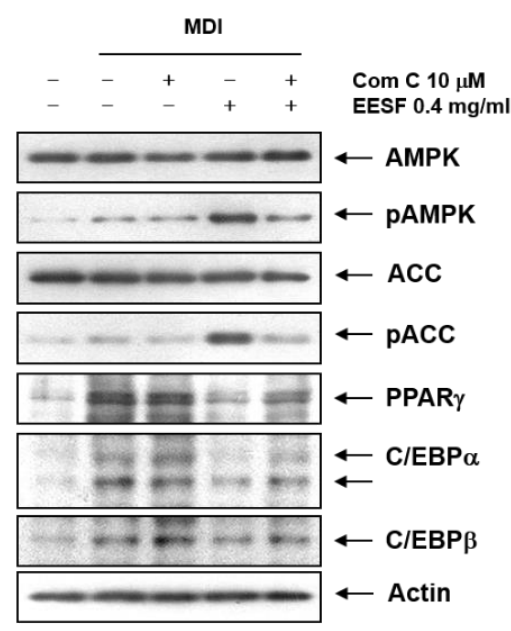

Figure 5. Role of the AMPK signaling pathway in the EESF-induced inhibition of adipogenesis in differentiated mouse 3T3-L1 adipocytes. (A) Effects of EESF on AMPK and ACC phosphorylation in 3T3-L1 cells. Confluent cells were treated with the indicated concentrations of EESF. (B) Effects of the AMPK inhibitor, Compound C, on the levels of adipogenic transcription factors in 3T3-L1 cells. Cells were pre-treated with compound $\mathrm{C}$ for $1 \mathrm{~h}$, and then treated with $100 \mathrm{~g} / \mathrm{mL}$ EESF. On day 8 , completely differentiated cells were lysed and cellular proteins were separated by SDSpolyacrylamide gels and transferred onto nitrocellulose membranes. The membranes were probed with the indicated antibodies. Proteins were visualized using an ECL detection system. Actin was used as an internal control.

\section{Conclusions}

In conclusion, our results indicate that EESF reduced adipocyte differentiation and adipogenesis in the 3T3-L1 pre-adipocytes by suppressing the adipogenic transcriptional factors and their downstream target genes without cytotoxicity. Further, EESF also increased the phosphorylation of AMPK and ACC; however, the artificial blockage of AMPK activity suppressed the inhibitory effects of EESF on the expressions of adipogenic transcriptional factors, demonstrating that EESF has significant anti-adipogenic effect that function via the AMP-activated protein kinase pathway. These findings suggested the possible use of EESF as a therapeutic substance or as a lead in the development of therapeutic substances for the prevention and management of obesity.

Author Contributions: Y.H.C. and J.-W.J. conceptualized the study, supervised experiments, supported the study, and wrote the paper. J.-W.J. partly supported financial funding and set up the experimental plans with writing manuscript. C.P. helped design and perform experiments. Y.-K.L., C.H.K., and K.-M.C. helped with various experiments. I.-J.Y. and G.-Y.K. shared reagents and helped in editing the paper. All authors have read and agreed to the published version of the manuscript.

Funding: This work was supported by a grant from the Nakdonggang National Institute of Biological Resources (NNIBR), funded by the Ministry of Environment (MOE) of the Republic of Korea (NNIBR2021021010).

Conflicts of Interest: The authors declare no conflict of interest. 


\section{References}

1. Attie, A.D.; Scherer, P.E. Adipocyte metabolism and obesity. J. Lipid Res. 2009, 50, S395-399.

2. Furukawa, S.; Fujita, T.; Shimabukuro, M.; Iwaki, M.; Yamada, Y.; Nakajima, Y.; Nakayama, O.; Makishima, M.; Matsuda, M.; Shimomura, I. Increased oxidative stress in obesity and its impact on metabolic syndrome. J. Clin. Invest. 2004, 114, 1752-1761.

3. Spiegelman, B.M.; Flier, J.S. Obesity and the regulation of energy balance. Cell 2001, 104, 531-543.

4. Popkin, B.M.; Adair, L.S.; Ng, S.W. Global nutrition transition and the pandemic of obesity in developing countries. Nutr. Rev. 2012, 70, 3-21.

5. Williams, E.P.; Mesidor, M.; Winters, K.; Dubbert, P.M.; Wyatt, S.B. Overweight and obesity: prevalence, consequences, and causes of a growing public health problem. Curr. Obes. Rep. 2015, 4, 363-370.

6. Derosa, G.; Cicero, A.F.; Murdolo, G.; Piccinni, M.N.; Fogari, E.; Bertone, G.; Ciccarelli, L.; Fogari, R. Efficacy and safety comparative evaluation of orlistat and sibutramine treatment in hypertensive obese patients. Diabetes Obes. Metab. 2005, 7, 47-55.

7. Esposito, P.; Rampino, T.; Gregorini, M.; Soccio, G.; Piotti, G.; Bedino, G.; Balenzano, C.T.; Roscini, E.; Cosmai, L.; Portalupi, V.; Libetta, C.; Dal Canton, A. Severe symptomatic hyponatremia during sibutramine therapy: a case report. Am. J. Kidney Dis. 2008, 52, 137-139.

8. Gesta, S.; Tseng, Y.H.; Kahn, C.R. Developmental origin of fat: tracking obesity to its source. Cell 2007, 131, 242-256.

9. Siersbæk, R.; Baek, S.; Rabiee, A.; Nielsen, R.; Traynor, S.; Clark, N.; Sandelin, A.; Jensen, O.N.; Sung, M.H.; Hager, G.L.; Mandrup, S. Molecular architecture of transcription factor hotspots in early adipogenesis. Cell Rep. 2014, 7, 1434-1442.

10. White, U.A.; Stephens, J.M. Transcriptional factors that promote formation of white adipose tissue. Mol. Cell Endocrinol. 2010, 318, 10-14.

11. Ji, S.; Doumit, M.E.; Hill, R.A. Regulation of Adipogenesis and Key Adipogenic Gene Expression by 1, 25Dihydroxyvitamin D in 3T3-L1 Cells. PLoS One 2015, 10, e0126142.

12. Yuan, H.D.; Piao, G.C. An active part of Artemisia sacrorum Ledeb. inhibits adipogenesis via the AMPK signaling pathway in 3T3-L1 adipocytes. Int. J. Mol. Med. 2011, 27, 531-536.

13. Chung D.K.; Lee S.I. Comparison of pharmacological effects of sophorae and sophorae fructus. Oriental Medicine 1985, 10, 5-18.

14. Joo, S.S.; Kang, H.C.; Lee, M.W.; Choi, Y.W.; Lee, D.I. Inhibition of IL-1beta and IL-6 in osteoblast-like cell by isoflavones extracted from Sophorae fructus. Arch. Pharm. Res. 2003, 26, 1029-1035.

15. Joo, S.S.; Won, T.J.; Kang, H.C.; Lee, D.I. Isoflavones extracted from Sophorae fructus upregulate IGF-I and TGF-beta and inhibit osteoclastogenesis in rat bone marrow cells. Arch. Pharm. Res. 2004, 27, 99-105.

16. Shim, J.G.; Yeom, S.H.; Kim, H.J.; Choi, Y.W.; Lee, D.I.; Song, K.Y.; Kwon, S.H.; Lee, M.W. Bone loss preventing effect of Sophorae Fructus on ovariectomized rats. Arch. Pharm. Res. 2005, 28, 106-110.

17. Joo, S.S.; Kwon, S.H.; Hwang, K.W.; Lee, D.I. Improvement of menopausal signs by isoflavones derived from Sophorae fructus in ovariectomized female rats and the antioxidant potentials in BV2 cells. Arch. Pharm. Res. 2005, 28, 566-572.

18. Lau, F.Y.; Chui, C.H.; Gambari, R.; Kok, S.H.; Kan, K.L.; Cheng, G.Y.; Wong, R.S.; Teo, I.T.; Cheng, C.H.; Wan, T.S.; Chan, A.S.; Tang, J.C. Antiproliferative and apoptosis-inducing activity of Brucea javanica extract on human carcinoma cells. Int. J. Mol. Med. 2005, 16, 1157-1162.

19. Lee, J.; Kim, K.W.; Kim, H.K.; Chae, S.W.; Jung, J.C.; Kwon, S.H.; Rheu, C.H. The effect of Rexflavone (Sophorae fructus extract) on menopausal symptoms in postmenopausal women: a randomized doubleblind placebo controlled clinical trial. Arch. Pharm. Res. 2010, 33, 523-530.

20. Gan, T.; Liu, Y.D.; Wang, Y.; Yang, J. Traditional Chinese Medicine herbs for stopping bleeding from haemorrhoids. Cochrane Database Syst. Rev. 2010, 6, CD006791.

21. Padilla-Benavides, T.; Velez-delValle, C.; Marsch-Moreno, M.; Castro-Muñozledo, F.; Kuri-Harcuch, W. Lipogenic enzymes complexes and cytoplasmic lipid droplet formation during adipogenesis. J. Cell Biochem. 2016, 117, 2315-2326.

22. Le Lay, S.; Dugail, I. Connecting lipid droplet biology and the metabolic syndrome. Prog. Lipid Res. 2009, 48, 191-195.

23. Yang, X.; Heckmann, B.L.; Zhang, X.; Smas, C.M.; Liu, J. Distinct mechanisms regulate ATGL-mediated adipocyte lipolysis by lipid droplet coat proteins. Mol. Endocrinol. 2013, 27, 116-126. 
24. Chen, H.C.; Farese, R.V. Jr. DGAT and triglyceride synthesis: a new target for obesity treatment? Trends Cardiovasc. Med. 2000, 10, 188-192.

25. Goldberg, I.J. Triglyceride: one molecule at the center of health and disease. Biochim. Biophys. Acta. 2012, 1821, 719-720.

26. Yano, T.; Kobori, S.; Sakai, M.; Anami, Y.; Matsumura, T.; Matsuda, H.; Kasho, M.; Shichiri, M. Beta-very low density lipoprotein induces triglyceride accumulation through receptor mediated endocytotic pathway in 3T3-L1 adipocytes. Atherosclerosis. 1997, 135, 57-64.

27. Lee, I.; Kim, J.; Ryoo, I.; Kim, Y.; Choo, S.; Yoo, I.; Min, B.; Na, M.; Hattori, M.; Bae, K. Lanostane triterpenes from Ganoderma lucidum suppress the adipogenesis in 3T3-L1 cells through down-regulation of SREBP-1c. Bioorg. Med. Chem. Lett. 2010, 20, 5577-5581.

28. Liang, Y.C.; Yang, M.T.; Lin, C.J.; Chang, C.L.; Yang, W.C. Bidens pilosa and its active compound inhibit adipogenesis and lipid accumulation via down-modulation of the C/EBP and PPAR $\gamma$ pathways. Sci. Rep. 2016, 6, 24285.

29. Dong, J.; Ishimori, N.; Paigen, B.; Tsutsui, H.; Fujii, S. Role of modulator recognition factor 2 in adipogenesis and leptin expression in 3T3-L1 cells. Biochem. Biophys. Res. Commun. 2008, 366, 551-555.

30. Evans, M.; Park, Y.; Pariza, M.; Curtis, L.; Kuebler, B.; McIntosh, M. Trans-10,cis-12 conjugated linoleic acid reduces triglyceride content while differentially affecting peroxisome proliferator activated receptor gamma2 and aP2 expression in 3T3-L1 preadipocytes. Lipids 2001, 36, 1223-1232.

31. Fisslthaler, B.; Fleming, I. Activation and signaling by the AMP-activated protein kinase in endothelial cells. Circ. Res. 2009, 105, 114-127.

32. Shen, Q.W.; Zhu, M.J.; Tong, J.; Ren, J.; Du, M. Ca ${ }^{2+} /$ calmodulin-dependent protein kinase kinase is involved in AMP-activated protein kinase activation by alpha-lipoic acid in C2C12 myotubes. Am. J. Physiol. Cell Physiol. 2007, 293, C1395-1403.

33. He, Y.; Li, Y.; Zhao, T.; Wang, Y.; Sun, C. Ursolic acid inhibits adipogenesis in 3T3-L1 adipocytes through LKB1/AMPK pathway. PLoS One 2013, 8, e70135.

34. Mitsuhashi, K.; Senmaru, T.; Fukuda, T.; Yamazaki, M.; Shinomiya, K.; Ueno, M.; Kinoshita, S.; Kitawaki, J.; Katsuyama, M.; Tsujikawa, M.; Obayashi, H.; Nakamura, N.; Fukui, M. Testosterone stimulates glucose uptake and GLUT4 translocation through LKB1/AMPK signaling in 3T3-L1 adipocytes. Endocrine 2016, 51, 174-184.

35. Pang, J.; Choi, Y.; Park, T. Ilex paraguariensis extract ameliorates obesity induced by high-fat diet: potential role of AMPK in the visceral adipose tissue. Arch. Biochem. Biophys. 2008, 476, 178-185. 